\title{
Genotyping of stathmin and its association with clinical factors and survival in patients with ovarian cancer
}

\author{
LISHA YING $^{1}$, DAN SU ${ }^{1}$, JIANQING ZHU ${ }^{1}$, SHENGLIN MA $^{1}$, DIONYSSIOS KATSAROS ${ }^{2}$ and HERBERT YU ${ }^{3}$ \\ ${ }^{1}$ Cancer Research Institute, Zhejiang Cancer Hospital, Hangzhou, Zhejiang, P.R. China; \\ ${ }^{2}$ Department of Obstetrics and Gynecology, Gynecologic Oncology and Breast Cancer Unit, University of Turin, Turin, Italy; \\ ${ }^{3}$ Cancer Epidemiology, Cancer Control and Population Sciences, Cancer Center, University of Hawaii, Hawaii, USA
}

Received October 15, 2012; Accepted January 8, 2013

DOI: $10.3892 / \mathrm{ol} .2013 .1144$

\begin{abstract}
Stathmin is closely correlated with the progression and prognosis of a number of types of human cancer. The present study analyzed the associations between genetic variations in the stathmin gene and clinical outcomes of ovarian cancer. A total of 178 patients with epithelial ovarian cancer were treated with cytoreductive surgery followed by platinum-based chemotherapy. DNA was extracted from fresh tumor samples obtained during surgery. A total of 32 DNA samples were selected randomly for resequencing of the stathmin gene. Tag single nucleotide polymorphisms (SNPs) were identified based on the haplotype model as analyzed by PolyPhred software. Direct sequencing was employed in the genotyping of stathmin in 178 cases. A total of 10 nucleotide variations in stathmin were identified, of which 3 high-frequency variations were known SNPs from databases and 7 were new variations with low frequencies. The tag SNPs rs159531 and rs11376635 were selected from the linkage disequilibrium block of the gene to genotype stathmin in 178 cases. The distribution of the rs159531 genotype in ovarian cancer was $52.8 \% \mathrm{C} / \mathrm{C}$, $35.4 \% \mathrm{C} / \mathrm{T}$ and $11.2 \% \mathrm{~T} / \mathrm{T}$. The distribution of the rs11376635 genotype in ovarian cancer was $32.0 \% \mathrm{G} / \mathrm{G}, 48.3 \% \mathrm{G} /-, 18.5 \%$ -/-. The main haplotypes calculated by phase 2.0 software were $55.6 \%$ CG, $27.8 \%$ T-, $15.4 \%$ C- and 1.2\% TG. However, no associations between the stathmin genotype or haplotype and the outcomes in patients with ovarian cancer were observed. The stathmin genotype and haplotype were not associated with the phenotype of patients with ovarian cancer.
\end{abstract}

\section{Introduction}

Epithelial ovarian cancer is the leading cause of mortality from gynecological cancer worldwide and the 5-year survival

Correspondence to: Dr Dan Su, Cancer Research Institute, Zhejiang Cancer Hospital, 38 Guangji Road, Hangzhou 310022, Zhejiang, P.R. China

E-mail:dan.su08@gmail.com

Key words: stathmin, genetic polymorphisms, ovarian cancer, genotype, haplotype rate remains $<40 \%$ despite good initial responses to standard post-operative adjuvant chemotherapy with combined paclitaxel and cisplatin (1-3). The majority of patients succumb to tumor recurrence or metastasis due to intrinsic or acquired drug resistance (4). A number of studies have demonstrated that variations in the genes for DNA repair, multi-drug resistance and drug-metabolizing enzymes were associated with the treatment response or prognosis of ovarian cancer (5-7).

Stathmin is encoded by the human STMN1 gene located at chromosome $1 \mathrm{p} 36.1$. The encoded protein prevents the assembly and promotes the disassembly of microtubules and is involved in the regulation of the microtubule filament system by destabilizing microtubules (8). Numerous studies have noted that upregulated expression of stathmin in several types of cancer, including breast (9), colorectal (10), endometrial (11), head and neck (12), hepatocarcinoma (13), lung (14), ovarian (15) and prostate cancer (16), was correlated with the malignant biological behavior of cancer cells, as well as poor treatment responses to microtubule-targeting drugs $(13,17,18)$. Our previous study also showed that high stathmin expression predicted an unfavorable prognosis in patients with ovarian cancer who received paclitaxel and platinum chemotherapy, supporting the possibility that stathmin may interfere with paclitaxel treatment, leading to a poor prognosis (19). However, few studies have reported the association between the stathmin genotype and treatment response or outcomes in patients with ovarian cancer. Moreover, the distribution, frequency and function of the variations of stathmin are not clear.

In the present study, the stathmin gene was resequenced by genotyping tag single nucleotide polymorphisms (SNPs) based on haplotype model analysis, then the associations of the stathmin genotype with treatment responses and disease progression were analyzed.

\section{Materials and methods}

Patients. A clinical study of ovarian cancer was conducted at the Gynecologic Oncology Unit at University of Turin (Turin, Italy) between October 1991 and February 2000. An ethics review committee at the university approved the study and all participants provided informed consent. From the study, 178 patients who had primary epithelial ovarian cancer and received post-operative platinum-based chemotherapy were 
Table I. Sathmin primer sequences for PCR.

\begin{tabular}{rll}
\hline No. & \multicolumn{1}{c}{ Forward primer } & \multicolumn{1}{c}{ Reverse primer } \\
\hline 1 & AATGCCGCAACAAGCATATTT & CAGAGCAGCACTGGGTTCTTT \\
2 & CAATTTCCTTGTGCCTTGC & GGTCCTTCCCACCTTCCAA \\
3 & ACTGCTCTGTCCGAGTGCTG & CCCGAGCCACACACAAAG \\
4 & GCTGAGGCCAGCAAGAGG & GGTCCAATCCGGGTAACTCC \\
5 & GAACTGTGAAGGGGGTGGTG & GTCTGTGTCTGACGTGGTGGA \\
6 & TGTTGGGCAAGGAAGCTTAAA & CAAATCAAAGGCGAAGACCTG \\
7 & TTCACCATGGCTTCTTCTGGT & GGGCTGATGAGGAAAGTTGTG \\
8 & TTGCCTGCAAATACATCTTCC & GGCAACACCATGTATTAAAGGAGA \\
9 & TGGAAGGAAATACCAGTCCTCA & TGTAAGCACTGAGGCTCTTCG \\
10 & TGATTGTGTTGCTCAGCTGGT & TTGCGAGTGGCACTTTTATTG \\
11 & TTTACAATGAGCTAGTTTTCTTTGG & CAGCTTCATGGGACTGGAAAA \\
12 & CCACACCCAGCCTGAATACAT & CAGTCTCGTCAGCAGGGTCTT \\
13 & GTTGTGTTGGGCCTCTTTGAG & GAGGGGCTCTATGGCTTGATT \\
14 & CAATCCCAATTCTGTCCCAAT & TGAGAGGCAAAGCACTGACAA \\
16 & TGACTCGGGTGGTTAAGGTTG & TATTTGCCCTACATGGGCGTA \\
\hline & TTCAACCAGAGGCTAATGAGTGA & ATGCATCCCCTTCAGTTTCCT \\
\hline
\end{tabular}

identified. The median age of the patients at surgery was 57.4 years, with a range of 26 to 82 years. Of the 178 patients, $33(18.5 \%)$ were diagnosed with stage I disease, $12(6.7 \%)$ were stage II, $120(67.4 \%)$ were stage III and 13 (7.3\%) were stage IV. The disease staging was classified according to the criteria of the International Federation of Gynecologists and Obstetricians (FIGO) (20). The histological type determined by following the World Health Organization (WHO) criteria (21) included serous, endometrioid, mucinous, clear cell and other epithelial tumors. For the data analysis, the tumor histotypes were grouped into serous $(43.3 \%, \mathrm{n}=77)$ and non-serous $(56.7 \%, \mathrm{n}=101)$. The majority of patients $(68.0 \%$, $\mathrm{n}=121$ ) had grade 3 tumors (poorly differentiated) and a small number had grade $2(19.1 \%, n=34)$ and grade $1(12.9 \%, n=23)$. When evaluated following cytoreduction, $108(60.7 \%)$ patients had residual lesions and $70(39.3 \%)$ patients had no residual lesions.

Following surgery, all the patients were treated with platinum-based chemotherapy; among them, 75 received platinum with paclitaxel $(42.1 \%)$, while 103 received platinum without paclitaxel $(57.9 \%)$. At 1 month after the chemotherapy, the treatment response was assessed based on the WHO criteria (22). A complete response (CR) required the complete disappearance of all measurable lesions, while a partial response (PR) had at least a $50 \%$ reduction in the measurable lesions. Stable disease (SD) was assigned to patients with a $<50 \%$ decrease or a $\leq 25 \%$ increase in the size of the measurable lesions, while progressive disease (PD) was assigned when lesions increased by $>25 \%$ or new lesions appeared. For non-measurable disease, the progression was defined as a doubling of CA-125 from the upper normal limit (23). For the data analysis, PR, SD and PD were grouped together as poor responders and were compared with CR. Of the 178 patients in the study, $71.9 \%(n=128)$ had a CR to treatment and $27.0 \%(n=48)$ had poor responses to treatment, which included 36 with PR, 4 with SD and 8 with $\mathrm{PD}$, while 2 patients (1.1\%) lacked treatment response data.
Table II. Sathmin primer sequences for sequencing.

\begin{aligned} & \hline No. \multicolumn{1}{c}{ Sequencing primer } \\ & \hline 1 AATGCCGCAACAAGCATATTT \\ & 2 GGTCCTTCCCTACCTTTCCAA \\ & 3 CCCGAGCCACACACAAAG \\ & 4 GGTCCAATCCGGGTAACTCC \\ & 5 GTCTGTGTCTGACGTGGTGGA \\ & 6 CAAATCAAAGGCGAAGACCTG \\ & 7 TTCACCATGGCTTCTTCTGGT \\ & 8 GGCAACACCATGTATTAAAGGAGA \\ & 9 TGTAAGCACTGAGGCTCTTCG \\ & 10 TTGCGAGTGGCACTTTTATTG \\ & 11 CAGCTTCATGGGACTGGAAAA \\ & 12 CCACACCCAGCCTGAATACAT \\ & 13 GAGGGGCTCTATGGCTTGATT \\ & 14 TGAGAGGCAAAGCACTGACAA \\ & 15 TATTTGCCCTACATGGGCGTA \\ & 16 ATGCATCCCCTTCAGTTTCCT \\ & \hline\end{aligned}

Resequencing and haplotype construction of stathmin. Genomic DNA was extracted from tumor samples following manual homogenization using the QIAamp1 DNA Mini kit (Qiagen, Valencia, CA, USA). A total of 32 DNA samples were randomly selected for resequencing from $1 \mathrm{kbp}$ upstream to $1 \mathrm{kbp}$ downstream of the stathmin gene. The entire coding exon and the flanking intronic sequences of the stathmin gene, covering $8.7 \mathrm{kbp}$, were amplified by PCR using the 16 pairs of primers designed by the online Primer3 software (http://primer3.wi.mit.edu/). The primer sequences for PCR are shown in Table I. A 20- $\mu 1$ mixture was prepared for each reaction and included $1 \mathrm{X}$ HotStarTaq buffer, 
Table III. Information of 10 DNA variations of stathmin.

\begin{tabular}{|c|c|c|c|c|c|c|}
\hline Name & $\begin{array}{c}\text { Position in } \\
\text { NC_000001.9 }\end{array}$ & $\begin{array}{l}\text { Reference } \\
\text { allele (A1) }\end{array}$ & $\begin{array}{c}\text { Other } \\
\text { allele (A2) }\end{array}$ & $\begin{array}{l}\mathrm{A} 2 \\
(\%)\end{array}$ & $\begin{array}{l}\text { Variation } \\
\text { property }\end{array}$ & $\begin{array}{c}\text { Nucleotide } \\
\text { variation }\end{array}$ \\
\hline Variation 1 & 26105623 & $\mathrm{C}$ & $\mathrm{T}$ & 3.1 & INTRON1 & $\mathrm{C} / \mathrm{T}$ \\
\hline $\begin{array}{l}\text { Variation } 2 \\
(\mathrm{rs} 213641)\end{array}$ & 26104943 & G & $\mathrm{T}$ & 56.3 & INTRON1 & $\mathrm{G} / \mathrm{T}$ \\
\hline Variation 3 & 26103346 & $\mathrm{~T}$ & $\mathrm{C}$ & 6.2 & INTRON2 & $\mathrm{T} / \mathrm{C}$ \\
\hline Variation 4 & 26102107 & $\mathrm{C}$ & $\mathrm{T}$ & 3.1 & INTRON3 & $\mathrm{C} / \mathrm{T}$ \\
\hline $\begin{array}{l}\text { Variation } 5 \\
\text { (rs159531) }\end{array}$ & 26100996 & $\mathrm{~T}$ & $\mathrm{C}$ & 68.8 & INTRON3 & $\mathrm{T} / \mathrm{C}$ \\
\hline Variation 6 & 26100558 & $\mathrm{~T}$ & $\mathrm{C}$ & 6.2 & INTRON4 & $\mathrm{T} / \mathrm{C}$ \\
\hline Variation 7 & $26100349^{\wedge} 26100360$ & $\mathrm{~N}$ & GG & 3.1 & INTRON4 & $\mathrm{N} / \mathrm{GG}^{\mathrm{a}}$ \\
\hline Variation 8 & 26100236 & G & $\mathrm{T}$ & 3.1 & INTRON4 & $\mathrm{G} / \mathrm{T}$ \\
\hline Variation 9 & 26098752 & A & $\mathrm{T}$ & 3.1 & 3'UTR & $\mathrm{A} / \mathrm{T}$ \\
\hline $\begin{array}{l}\text { Variation } 10 \\
\text { (rs11376635) }\end{array}$ & $26098388^{\wedge} 26098389$ & - & $\mathrm{G}$ & 56.3 & 3'UTR & $-/ G$ \\
\hline
\end{tabular}

${ }^{\mathrm{a}} \mathrm{N}=$ tgttaggttct.

$2.0 \mathrm{mM} \mathrm{Mg}{ }^{2+}, 0.2 \mathrm{mM}$ dNTP, $0.2 \mu \mathrm{M}$ of each primer, 1 unit HotStarTaq polymerase (Qiagen) and $1 \mu 1$ template DNA (5-10 ng/ $/ \mu \mathrm{l})$. The cycling program was $95^{\circ} \mathrm{C}$ for $15 \mathrm{~min} ; 11$ cycles of $94^{\circ} \mathrm{C}$ for $15 \mathrm{sec}, 62-0.5^{\circ} \mathrm{C}$ per cycle for $40 \mathrm{sec}, 72^{\circ} \mathrm{C}$ for $1 \mathrm{~min} ; 24$ cycles of $94^{\circ} \mathrm{C}$ for $15 \mathrm{sec}, 56^{\circ} \mathrm{C}$ for $30 \mathrm{sec}, 72^{\circ} \mathrm{C}$ for $1 \mathrm{~min}$; and $72^{\circ} \mathrm{C}$ for $2 \mathrm{~min}$. The PCR products were purified with shrimp alkaline phosphatase enzyme (SAP; Promega, Madison, WI, USA) and exonuclease I (Exo I; Epicentre, Madison, WI, USA). Cycle sequencing was performed with the BigDye Terminator Cycle Sequencing Ready Reaction kit (Applied Biosystems, Foster City, CA, USA) on an ABI 3130 Genetic Analyzer (Applied Biosystems). The primer sequences for sequencing are shown in Table II. SNPs were identified by the PolyPhred program (http://droog.mbt.washington.edu/poly_get.html). Pairwise linkage disequilibrium (LD) between the sequence variations was analyzed by two parameters, ID'l and $r^{2}$. The haplotypes from the sequence variations were reconstructed with the PHASE 2.1 program (http://www.stat.washington. edu/stephens/phase/download.html).

SNP genotyping in 178 ovarian cancer tissue samples. Direct sequencing in 178 patients with ovarian cancer was used to genotype 2 tag SNPs with high allelic frequency identified according to the haplotype model. The method of sequencing was as mentioned previously.

Statistical analysis. The frequencies of various genotypes and haplotypes were compared between patients with and without treatment responses using the Chi-square test. Multivariate analyses were performed with the use of unconditional logistic regression analysis to assess the associations between the treatment responses and each genetic polymorphism while adjusting for patient age at diagnosis, tumor histology, disease stage, grade and residual tumor. The odds ratios (ORs) and corresponding $95 \%$ confidence intervals (CIs) were calcu- lated using the logistic regression model. By combining the data of stathmin expression from our previous study (19), the associations between the phenotype and genotype of stathmin were analyzed using Pearson's correlation test. All P-values reported were two-sided and $\mathrm{P} \leq 0.05$ was considered to indicate statistically significant differences. The statistical analysis was performed using SPSS software, Version 11.0 (SPSS Inc., Chicago, IL, USA).

\section{Results}

Variations in the stathmin gene. As shown in Table III, 10 variations in stathmin were identified, including 2 insertion/deletion and 8 single nucleotide variations, of which 3 high-frequency variations were known SNPs from the dbSNP database of the NCBI website, rs213641 (G/T) in intron 1, rs159531 (T/C) in intron 3 and rs11376635 (-/G) separately in the 3'UTR. The distributions of these SNPs in 32 samples were similar to the data from the dbSNP database. Of the variations, 7 were reported for the first time and all were present at low frequencies.

Tag SNP and haplotype in stathmin. The analysis of pairwise LD and $r^{2}$ showed these 10 variation loci were in high LD and the entire gene was in an LD block. Based on parameters including $\mathrm{r}^{2}>0.8$ and allelic frequency $>10 \%, 2$ tag SNPs were identified, rs159531 (T/C) and rs11376635 (-/G). According to the 2 tag SNPs, the main haplotypes were calculated as CG, $\mathrm{T}$ - and C-.

Genotyping of stathmin in 178 patients with ovarian cancer. Using direct sequencing in 178 patients with ovarian cancer, 2 tag SNPs with high allelic frequency, rs159531 (G/T) and rs11376635 (T/C), were identified for genotyping. The genotypes of rs159531 were $52.8 \% \mathrm{C} / \mathrm{C}$ (94/178), 35.4\% C/T (63/178), $11.2 \% \mathrm{~T} / \mathrm{T}(20 / 178)$ and $0.6 \%$ missing (1/178). The 
Table IV. Patient clinicopathological characteristics and genotypes.

\begin{tabular}{|c|c|}
\hline Variable & Value \\
\hline Age (years), median (range) & $57.4(26-82)$ \\
\hline \multicolumn{2}{|l|}{ Stage, n (\%) } \\
\hline I-II & $45(25.3)$ \\
\hline III-IV & $133(74.7)$ \\
\hline \multicolumn{2}{|l|}{ Grade, n (\%) } \\
\hline $1-2$ & $57(32.0)$ \\
\hline 3 & $121(68.0)$ \\
\hline \multicolumn{2}{|l|}{ Histology, n (\%) } \\
\hline Non-serous & $101(56.7)$ \\
\hline Serous & $77(43.3)$ \\
\hline \multicolumn{2}{|l|}{ Residual tumor, n (\%) } \\
\hline No residual & $70(39.3)$ \\
\hline Residual & $108(60.7)$ \\
\hline \multicolumn{2}{|l|}{ Treatment response, $\mathrm{n}(\%)$} \\
\hline Complete response & $128(71.9)$ \\
\hline Poor response & $48(27.0)$ \\
\hline Missing & $2(1.1)$ \\
\hline \multicolumn{2}{|l|}{ Chemotherapy, n (\%) } \\
\hline Platinum with paclitaxel & $75(42.1)$ \\
\hline Platinum without paclitaxel & $103(57.9)$ \\
\hline \multicolumn{2}{|l|}{ rs159531, n (\%) } \\
\hline $\mathrm{C} / \mathrm{C}$ & $94(52.8)$ \\
\hline $\mathrm{C} / \mathrm{T}$ & $63(35.4)$ \\
\hline $\mathrm{T} / \mathrm{T}$ & $20(11.2)$ \\
\hline Missing & $1(0.6)$ \\
\hline \multicolumn{2}{|l|}{ rs11376635, n (\%) } \\
\hline $\mathrm{G} / \mathrm{G}$ & $57(32.0)$ \\
\hline $\mathrm{G} /-$ & $86(48.3)$ \\
\hline$-/-$ & $33(18.5)$ \\
\hline Missing & $2(1.1)$ \\
\hline \multicolumn{2}{|l|}{ Haplotype, n (\%) } \\
\hline $\mathrm{C} / \mathrm{G}$ & $142(79.8)$ \\
\hline $\mathrm{T} /-$ & $17(9.6)$ \\
\hline $\mathrm{C} /-$ & $16(9)$ \\
\hline $\mathrm{T} / \mathrm{G}$ & $3(1.7)$ \\
\hline
\end{tabular}

genotypes of rs11376635 were $32 \% \mathrm{G} / \mathrm{G}$ (57/178), $48.3 \%$ G/- (86/178), $18.5 \%$-/- (33/178) and 1.1\% missing (2/178). The distribution of the main haplotypes were $79.8 \% \mathrm{CG}$ (142/178), 9.6\% T- (17/178), 9.0\% C- (16/178) and $1.7 \%$ TG (3/178; Table IV).

Correlation of the genotypes of stathmin and clinical characteristics of ovarian cancer. As shown in Table V, the genotypes or haplotypes of stathmin were not observed to be associated with disease stage, tumor grade, histology, residual tumor size or treatment response in 178 patients in ovarian cancer.
No significant differences in the survival of patients with ovarian cancer according to the genotypes of stathmin were observed.

Association of genotype and phenotype in stathmin. By combining the present data with our previous mRNA expression of stathmin data, no significant correlations between the genotype and phenotype of stathmin were observed, as shown in Table VI.

\section{Discussion}

Stathmin, first identified as a cytosolic phosphoprotein in neuroendocrine cells (24), has been observed to be closely correlated with the progression and prognosis of a number of types of human cancer. High expression levels of stathmin indicate increased proliferation, invasion and poor prognosis (25). However, few studies have reported the somatic or genetic variants of the stathmin gene. In the present study, 10 nucleotide variations of stathmin, including 3 SNP sites with high frequency from the dbSNP database of the NCBI website and 7 new variations with low frequencies, were identified by resequencing. All were non-coding variations, 2 located in 3'UTR and the others in introns. Variations in the 3'UTR may have significant functional implications for miRNA binding and posttranscriptional regulation and be associated with human diseases $(26,27)$. Additionally, increasing evidence indicates that genomic variants in non-coding sequences may have unexpected deleterious effects on the splicing of the gene transcript (28).

In order to observe the possible biological function of those variations, 2 tag SNPs with high allelic frequency (rs159531 and rs11376635) were selected for genotyping and the associations between the genetic variants of stathmin and clinical characteristics of 178 patients with ovarian cancer were analyzed. The genotypic frequencies of these 2 tag SNPs were similar to the data from the dbSNP database. The majority of patients $(79.8 \%, 142 / 178)$ had the CG haplotype. However, the genotype of the tag SNP or haplotype was not associated with the expression level of stathmin and no significant associations among genotype, clinical characteristics and outcomes in patients with ovarian cancer were observed.

Stathmin is a microtubule-destabilizing protein that regulates microtubule dynamics by preventing tubulin polymerization and promoting microtubule disassembly during cell cycle progression $(29,30)$. Changes to the mRNA levels of the stathmin gene may disturb microtubule stabilization and thereby affect the treatment response in cancer therapy, particularly in microtubule-targeting drugs. A large number of studies have reported that the overexpression of stathmin may be an independent predictor of poor treatment responses or worse prognoses and a potential target in numerous types of cancer (31-34). We hypothesized that genetic variation in stathmin is associated with upregulated expression of stathmin which may affect the outcome of patients with ovarian cancer. However, no significant associations were observed in the present study. To the best of our knowledge, no study has shown germline or somatic variations of stathmin in cancer, although certain studies 
Table V. Correlation of the genotype of stathmin with the clinical characteristics of ovarian cancer.

\begin{tabular}{|c|c|c|c|c|c|c|c|c|}
\hline \multirow[b]{2}{*}{ Variable } & \multicolumn{3}{|c|}{ rs159531, n (\%) } & \multicolumn{3}{|c|}{ rs11376635, n (\%) } & \multicolumn{2}{|c|}{ Haplotype, n (\%) } \\
\hline & $\mathrm{C} / \mathrm{C}$ & $\mathrm{C} / \mathrm{T}$ & $\mathrm{T} / \mathrm{T}$ & $\mathrm{G} / \mathrm{G}$ & G/- & $-/-$ & $\mathrm{C} / \mathrm{G}$ & $\mathrm{T} /-, \mathrm{C} /-, \mathrm{T} / \mathrm{G}$ \\
\hline \multicolumn{9}{|l|}{ Stage } \\
\hline I-II & $24(25.5)$ & $18(28.6)$ & $3(15.0)$ & $15(26.3)$ & $20(23.3)$ & $10(30.3)$ & $35(24.6)$ & $10(27.8)$ \\
\hline III-IV & $70(74.5)$ & $45(71.4)$ & $17(85.0)$ & $42(73.7)$ & $66(76.7)$ & $23(69.7)$ & $107(75.4)$ & $26(72.2)$ \\
\hline P-value & 0.478 & - & - & 0.724 & - & - & 0.674 & - \\
\hline \multicolumn{9}{|l|}{ Grade } \\
\hline $1-2$ & $32(34.0)$ & $17(27.0)$ & $8(40.0)$ & $19(33.3)$ & $26(30.2)$ & $12(36.4)$ & $45(31.7)$ & $12(33.3)$ \\
\hline 3 & $62(66.0)$ & $46(73.0)$ & $12(60.0)$ & $38(66.7)$ & $60(69.8)$ & $21(63.6)$ & $97(68.3)$ & $24(66.7)$ \\
\hline P-value & 0.475 & - & - & 0.801 & - & - & 0.844 & - \\
\hline \multicolumn{9}{|l|}{ Histology } \\
\hline Non-Serous & $57(60.0)$ & $32(50.8)$ & $11(55.0)$ & $32(56.1)$ & $47(54.7)$ & $20(60.6)$ & $80(56.3)$ & $21(58.3)$ \\
\hline Serous & $37(39.4)$ & $31(49.2)$ & $9(45.0)$ & $25(43.9)$ & $39(45.3)$ & $13(39.4)$ & $62(43.7)$ & $15(41.7)$ \\
\hline P-value & 0.47 & - & - & 0.842 & - & - & 0.853 & - \\
\hline \multicolumn{9}{|l|}{ Residual tumor } \\
\hline No residual & $41(43.6)$ & $24(38.1)$ & $5(25.0)$ & $28(49.1)$ & $31(36.0)$ & $11(33.3)$ & $59(41.5)$ & $11(30.6)$ \\
\hline Residual & $53(56.4)$ & 39 (61.9) & $15(75.0)$ & $29(50.9)$ & $55(64.0)$ & $22(66.7)$ & $83(58.5)$ & $25(69.4)$ \\
\hline P-value & 0.29 & - & - & 0.207 & - & - & 0.256 & - \\
\hline \multicolumn{9}{|l|}{ Treatment response } \\
\hline Complete responder & $67(72.8)$ & $45(71.4)$ & $15(75.0)$ & $40(72.7)$ & $63(73.3)$ & $23(69.7)$ & $102(72.9)$ & $26(72.2)$ \\
\hline Poor responder & $25(27.2)$ & $18(28.6)$ & $5(25.0)$ & $15(27.3)$ & $23(26.7)$ & $10(30.3)$ & $38(27.1)$ & $10(27.8)$ \\
\hline P-value & 0.95 & - & - & 0.925 & - & - & 1.000 & - \\
\hline
\end{tabular}

Table VI. Correlation of genotype, haplotype and stathmin expression.

\begin{tabular}{|c|c|c|c|c|}
\hline & \multicolumn{3}{|c|}{ Stathmin expression $(\%)$} & \multirow[b]{2}{*}{ P-value } \\
\hline & Low & Median & High & \\
\hline \multicolumn{5}{|l|}{ rs 159531} \\
\hline $\mathrm{C} / \mathrm{C}$ & $33(35.1)$ & $29(30.9)$ & $32(34.0)$ & \multirow{3}{*}{0.960} \\
\hline $\mathrm{C} / \mathrm{T}$ & $19(30.2)$ & $22(34.9)$ & $22(34.9)$ & \\
\hline $\mathrm{T} / \mathrm{T}$ & $7(35.0)$ & $7(35.0)$ & $6(30.0)$ & \\
\hline \multicolumn{5}{|l|}{ rs 11376635} \\
\hline $\mathrm{G} / \mathrm{G}$ & $22(38.6)$ & $16(28.1)$ & $19(33.3)$ & \multirow{3}{*}{0.366} \\
\hline G/- & $23(26.7)$ & $30(34.9)$ & $33(38.4)$ & \\
\hline$-/-$ & $14(42.4)$ & $11(33.3)$ & $8(24.2)$ & \\
\hline \multicolumn{5}{|l|}{ Haplotype } \\
\hline $\mathrm{C} / \mathrm{G}$ & $44(31.0)$ & $46(32.4)$ & $52(36.6)$ & \multirow[t]{2}{*}{0.345} \\
\hline $\mathrm{T} /-, \mathrm{C} /-, \mathrm{T} / \mathrm{G}$ & $15(41.7)$ & $12(33.3)$ & $9(25.0)$ & \\
\hline
\end{tabular}

have reported that SNPs in stathmin were associated with the etiopathogenesis of a broad range of neuropsychiatric disorders with dysfunctional networking. For example, SNP rs182455, located in the promoter of stathmin and rs213641, an SNP in the 5'UTR of the alternatively transcribed exon $1 \mathrm{c}$ in stathmin, which may modify the binding of nerve growth factor-induced protein $\mathrm{C}$, were identified in fear and anxiety processing and cognitive and affective control processes $(35,36)$. By contrast, Buttmann et al also analyzed rs182455 in 647 clinically well-characterized multiple sclerosis (MS) patients and 519 healthy controls, but no associations of the genotype of rs182455 SNPs with MS susceptibility or clinical disease course were observed (3). Furthermore, rs12037513 and rs159522, both located in close vicinity to the stathmin gene, have no any association with schizophrenia (37). 
In conclusion, although no significant associations were observed between the genotype of stathmin and clinical characteristics or outcomes in patients with ovarian cancer in the present study, the results provided information concerning somatic or genetic variations of stathmin in patients with ovarian cancer.

\section{Acknowledgements}

The present study was supported by the Qianjiang Talent Project of Zhejiang Province of China (No. 2010R10060) and National Natural Science Young Foundation (No. 81101986).

\section{References}

1. Du Bois A, Lück HJ, Meier W, et al: Carboplatin plus paclitaxel as first-line chemotherapy in previously untreated advanced ovarian cancer. German AGO Study Group Ovarian Cancer. Arbeitsgemeinschaft Gynäkologische Onkologie. Semin Oncol 24 (4 Suppl 11): S11-28-S11-33, 1997.

2. Aravantinos G, Fountzilas G, Kosmidis P, et al: Paclitaxel plus carboplatin versus paclitaxel plus alternating carboplatin and cisplatin for initial treatment of advanced ovarian cancer: long-term efficacy results: a Hellenic Cooperative Oncology Group (HeCOG) study. Ann Oncol 16: 1116-1122, 2005.

3. Buttmann M, Nowak E, Kroner A, Hemmer B, Lesch KP and Rieckmann P: Analysis of the stathmin rs182455 single nucleotide promoter polymorphism in patients with multiple sclerosis. J Neurogenet 22: 181-186, 2008.

4. Lippert TH, Ruoff H and Volm M: Intrinsic and Acquired Drug Resistance in Malignant Tumors. The main reason for therapeutic failure. Arzneimittelforschung 58: 261-264, 2008.

5. Fleming ND, Agadjanian H, Nassanian H, et al: Xeroderma pigmentosum complementation group $\mathrm{C}$ single-nucleotide polymorphisms in the nucleotide excision repair pathway correlate with prolonged progression-free survival in advanced ovarian cancer. Cancer 118: 689-697, 2012.

6. Lose F, Nagle CM, O'Mara T, et al: Vascular endothelial growth factor gene polymorphisms and ovarian cancer survival. Gynecol Oncol 119: 479-483, 2010.

7. Quaye L, Gayther SA, Ramus SJ, et al: The effects of common genetic variants in oncogenes on ovarian cancer survival. Clin Cancer Res 14: 5833-5839, 2008.

8. Belmont LD and Mitchison TJ: Identification of a protein that interacts with tubulin dimers and increases the catastrophe rate of microtubules. Cell 84: 623-631, 1996.

9. Golouh R, Cufer T, Sadikov A, et al: The prognostic value of Stathmin-1, S100A2, and SYK proteins in ER-positive primary breast cancer patients treated with adjuvant tamoxifen monotherapy: an immunohistochemical study. Breast Cancer Res Treat 110: 317-326, 2008.

10. Zheng P, Liu YX, Chen L, et al: Stathmin, a new target of PRL-3 identified by proteomic methods, plays a key role in progression and metastasis of colorectal cancer. J Proteome Res 9: 4897-4905, 2010.

11. Trovik J, Wik E, Stefansson IM, et al: Stathmin overexpression identifies high-risk patients and lymph node metastasis in endometrial cancer. Clin Cancer Res 17: 3368-3377, 2011.

12. Canzonieri V, Barzan L, Franchin G, et al: Alteration of G1/S transition regulators influences recurrences in head and neck squamous carcinomas. J Cell Physiol 227: 233-238, 2012.

13. Singer S, Ehemann V, Brauckhoff A, et al: Protumorigenic overexpression of stathmin/Op18 by gain-of-function mutation in $\mathrm{p} 53$ in human hepatocarcinogenesis. Hepatology 46: 759-768, 2007.

14. Rosell R, Scagliotti G, Danenberg KD, et al: Transcripts in pretreatment biopsies from a three-arm randomized trial in metastatic non-small-cell lung cancer. Oncogene 22: 3548-3553, 2003.

15. Wei SH, Lin F, Wang X, Gao P and Zhang HZ: Prognostic significance of stathmin expression in correlation with metastasis and clinicopathological characteristics in human ovarian carcinoma. Acta Histochem 110: 59-65, 2008.
16. Ghosh R, Gu G, Tillman E, et al: Increased expression and differential phosphorylation of stathmin may promote prostate cancer progression. Prostate 67: 1038-1052, 2007.

17. Carr JR, Park HJ, Wang Z, Kiefer MM and Raychaudhuri P: FoxM1 mediates resistance to herceptin and paclitaxel. Cancer Res 70: 5054-5063, 2010.

18. Alli E, Yang JM and Hait WN: Silencing of stathmin induces tumor-suppressor function in breast cancer cell lines harboring mutant p53. Oncogene 26: 1003-1012, 2007.

19. Su D, Smith SM,Preti M, et al: Stathmin and tubulin expression and survival of ovarian cancer patients receiving platinum treatment with and without paclitaxel. Cancer 115: 2453-2463, 2009.

20. Shepherd JH: Revised FIGO staging for gynaecological cancer. Br J Obstet Gynaecol 96: 889-892, 1989.

21. Scully RE and Sobin LH: Histologic typing of ovarian tumors. Arch Pathol Lab Med 111: 794-795, 1987.

22. Miller AB, Hoogstraten B, Staquet M and Winkler A: Reporting results of cancer treatment. Cancer 47: 207-214, 1981.

23. Rustin GJ, Nelstrop AE, Tuxen MK and Lambert HE: Defining progression of ovarian carcinoma during follow-up according to CA 125: a North Thames Ovary Group Study. Ann Oncol 7: 361-364, 1996.

24. Sobel A and Tashjian AH Jr: Distinct patterns of cytoplasmic protein phosphorylation related to regulation of synthesis and release of prolactin by GH cells. J Biol Chem 258: 10312-10324, 1983.

25. Belletti B and Baldassarre G: Stathmin: a protein with many tasks. New biomarker and potential target in cancer. Expert Opin Ther Target 15: 1249-1266, 2011.

26. Saunders MA, Liang H and Li WH: Human polymorphism at microRNAs and microRNA target sites. Proc Natl Acad Sci USA 104: 3300-3305, 2007.

27. Sun G, Yan J, Noltner K, et al: SNPs in human miRNA genes affect biogenesis and function. RNA 15: 1640-1651, 2009.

28. Pagani F and Baralle FE: Genomic variants in exons and introns: identifying the splicing spoilers. Nat Rev Genet 5: 389-396, 2004.

29. Jourdain I, Lachkar S, CharbautE, et al: A synergistic relationship between three regions of stathmin family proteins is required for the formation of a stable complex with tubulin. Biochem J 378: 877-888, 2004.

30. Jourdain L, Curmi P, Sobel A, Pantaloni D and Carlier MF: Stathmin: a tubulin-sequestering protein which forms a ternary T2S complex with two tubulin molecules. Biochemistry 36 : 10817-10821, 1997

31. Oishi Y, Nagasaki K, Miyata S, et al: Functional pathway characterized by gene expression analysis of supraclavicular lymph node metastasis-positive breast cancer. J Hum Genet 52: 271-279, 2007.

32. Salvesen HB, Carter SL, Mannelqvist M, et al: Integrated genomic profiling of endometrial carcinoma associates aggressive tumors with indicators of PI3 kinase activation. Proc Natl Acad Sci USA 106: 4834-4839, 2009.

33. Yuan RH, Jeng YM, Chen HL, et al: Stathmin overexpression cooperates with p53 mutation and osteopontin overexpression, and is associated with tumour progression, early recurrence, and poor prognosis in hepatocellular carcinoma. J Pathol 209: $549-558,2006$

34. Meng XL, Su D, Wang L, et al: Low expression of stathmin in tumor predicts high response to neoadjuvant chemotherapy with docetaxel-containing regimens in locally advanced breast cancer. Genet Test Mol Biomarkers 16: 689-694, 2012.

35. Brocke B, Lesch KP, Armbruster D, et al: Stathmin, a gene regulating neural plasticity, affects fear and anxiety processing in humans. Am J Med Genet B Neuropsychiatr Genet 153B: 243-251, 2010.

36. Ehlis AC, Bauernschmitt K, Dresler T, et al: Influence of a genetic variant of the neuronal growth associated protein Stathmin 1 on cognitive and affective control processes: an event-related potential study. Am J Med Genet B Neuropsychiatr Genet 156B: 291-302, 2011.

37. Yamada K, Matsuzaki S, Hattori T, et al: Increased stathmin1 expression in the dentate gyrus of mice causes abnormal axonal arborizations. PLoS One 5: e8596, 2010. 